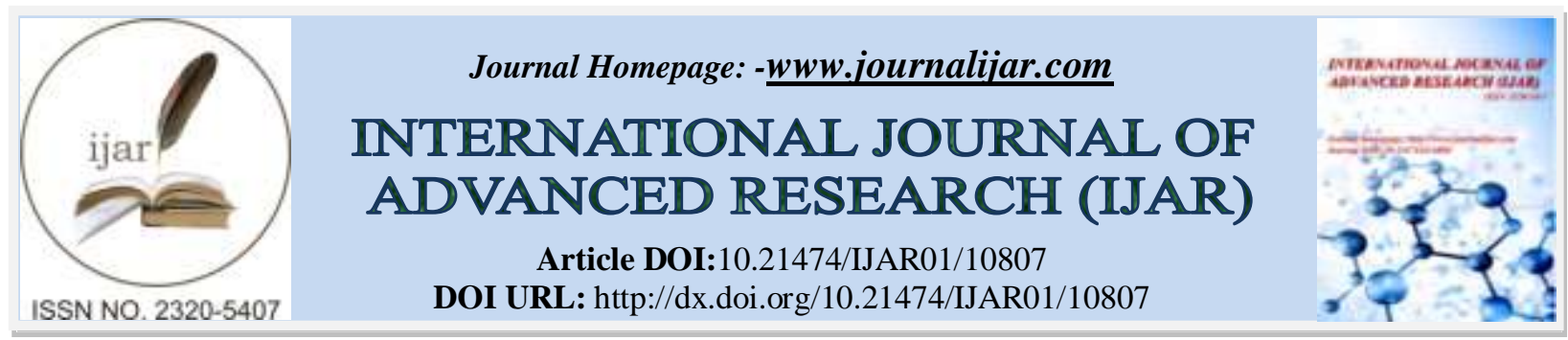

RESEARCH ARTICLE

\title{
LE SYNDROME DES ANTI-PHOSPHOLIPIDES OBSTETRICAL: EXPERIENCE DU SERVICE DE MEDECINE INTERNE DE MARRAKECH
}

\author{
M. Yahyaoui, H. Taouti, M. Zahlane and L. Essaadouni
}

Service De Médecine Interne CHU Mohammed VI, Marrakech.

\section{Manuscript Info}

Manuscript History

Received: 12 February 2020

Final Accepted: 14 March 2020

Published: April 2020

\begin{abstract}
The antiphospholipid syndrome (APS) or Hughes syndrome is a systemic autoimmune pathology of the young subject. It is a clinicobiological entity that associates clinical manifestations such as recurrent venous and arterial thrombosis and/or repetitive obstetric complications. Due to the persistent presence in the blood, at least 12 weeks apart, of antiphospholipid antibodies. Study design: This is a retrospective study over a period of 12 years of records of patients followed for APS in the Internal Medicine Department of the Mohammed VI University Hospital of Marrakech, aiming to establish the epidemiological, clinical, biological, therapeutic and evolution of these patients. Results: Fifty-four cases of APS were collected, the mean age at onset of the first clinical manifestations of the syndrome was 36 years, with a clear female predominance (sex ratio $\mathrm{F} / \mathrm{H}$ of 9.8). Thirty women $(55.5 \%)$ had obstetric complications dominated by fetal loss $(52 \%)$, followed by pre-eclampsia (13\%), premature delivery, eclampsia and HELLP syndrome. The APS was primary in $57.4 \%$ of cases and secondary in $42.6 \%$ of cases (including $40.7 \%$ of systemic lupus erythematosus). The catastrophic antiphospholipid syndrome was noted in 2 cases (3.7\%). Biologically, thrombocytopenia was found in $15 \%$ of cases and hemolytic anemia in $9.5 \%$ of cases. Immunologically, anticardiolipins, anti $\beta 2$ glycoproteins and lupus anticoagulant were positive in $74 \%, 61 \%$ of cases and $20.4 \%$ of cases, respectively. The persistence of antiphospholipid antibodies could not be verified in 11 patients. Therapeutically, all patients with thrombosis were treated with low molecular weight heparin (LMWH) and vitamin $\mathrm{K}$ antagonists (VKA). Anti-platelet agents were prescribed in $40.7 \%$ of cases. The evolution was favorable in most of the cases, 3 deaths were noted of which 2 patients had presented with a catastrophic antiphospholipid syndrome(CAPS).
\end{abstract}

Copy Right, IJAR, 2020, All rights reserved.

\section{Introduction:-}

Le syndrome des antiphospholipides (SAPL) ou syndrome de Hughes estunepathologie auto-immune systémique du sujetjeune. C'estuneentitéclinicobiologiquecaractérisée par un risqueaccru de phénomènesthrombotiqueset/ou de complications obstétricales dues à la présencepersistante dans le sang, à au moins 12 semainesd'intervalles, d'anticorpsantiphospholipides. 
Le SAPL obstétrical (OAPS) estdéfini quant à lui, par la présence de fausses couches consécutivesrépétées (au moins 3 épisodes à $<10$ semainesd'aménorrhée [SA]) et/ou de mortsfoetales ( $>10$ semainesd'aménorrhée) et/ou de naissances prématurées $(<34$ semainesd'aménorrhée) en rapport avec uneinsuffisanceplacentaire [1; 2].Il s'agitd'uneentité à part entière, dont le mécanismephysiopathologiqueseraitindividualisé de celui du SAPL vasculaire. Les complications obstétricalesseraient dues à un phénomèneinflammatoireciblant le placenta, sous l'action du système du complément, en plus de l'altération de l'invasiontrophoblastique et de l'angiogenèseendométriale [3].

\section{Patientes et Methods:-}

Nous avonsmenéune étude rétrospective sur unepériode de 12 ans, incluant les dossiers de patientessuivies pour SAPL avec manifestations obstétricaleshospitalisées au service de Médecine Interne du CHU Mohammed VI de Marrakech.

Le SAPL étaitdéfiniselon les critères de Sapporo révisés à Sydney en 2006 [2].

L'objectifétaitd'établir le profilépidémiologique, clinique, biologique, thérapeutique et évolutif de cespatientes.

Les variables étudiéescorrespondaient à des élémentsdémographiques (âge), cliniques (mode de révélation, présenceou non de thrombose associée, type des évènementsobstétricaux et des complications maternelles, association ou non à un lupus systémique, traitementinstauré et évolution) et biologiques(biologie antiphospholipide).

\section{Résultats:-}

Cinquante-quatre cas de cas de SAPL ontétécolligés, parmilesquels 34 patientes $(62,9 \%)$ présentaient des complications obstétricales.

L'incidencemoyenneétait de 3 cas par an (Figure 1)

L'âgemoyen au moment de l'apparition des premières manifestations cliniques du SAPL était de 36,76 ans, avec des intervallesde 22 à 57 ans.

Le SAPL étaitobstétricalpur dans 55,88 \% des cas (n=19), et associé à des manifestations vasculaires dans $44,11 \%$ des cas $(\mathrm{n}=15)$.Il étaitprimaire dans $61 \%$ des cas $(\mathrm{n}=21)$ et secondaire à un lupus systémique dans $38,23 \%$ des cas $(\mathrm{n}=13)$.

Les manifestations obstétricalesétaientrévélatrices chez 5 patientes $(14,70 \%)$, les autres manifestations cliniquessontrésumées dans le tableau 1.

Les manifestations obstétricalesétaientdominées par les pertesfotales $>10$ SA dans $76,45 \%$ des cas, suivies par les fausses couches $<10$ SA dans $38 \%$ La pré-eclampsieétaitprésenteans $14,7 \%$, le HELLP (HemolysisElevetedLiverLowPlatelet) syndrome n'étaitobservé que dans 2,9\% des cas (Figure 2).

Sur le plan biologique, l'anticorps anti-cardiolipineétaitl'anticorps le plus fréquemmentretrouvé, dans $82 \%$ des cas $(\mathrm{n}=28)$, suivi par l'antibêta 2 glycoprotéine dans $58,8 \%$ des cas $(\mathrm{n}=20)$ et l'anti-coagulant lupique circulant dans $8,82 \%$ des cas $(\mathrm{n}=3)$. Aucuncas de triple positivitén'aétéretrouvé. La confirmation de la positivité des antiphospholipides a puêtreréalisée dans $77 \%$ des cas. Leurcorrélation avec les évènementsobstétricauxestreprésentée dans la figure 3.

Sur le plan thérapeutique, toutes les patientesayantprésentéune thrombose systémiqueontreçuune anti-coagulation par héparine de bas poidsmoléculaire à dose curative (58\%), unecorticothérapie a étéinstaurée chez $38 \%$ des patientes, les anti-paludéens de synthèse chez $17 \%$ des cas et un traitement par immunosuppresseur a étéinitié chez $17 \%$ des cas, selon les atteintesviscérales du lupus systémique. Une association d'héparine à dose préventive avec de l'aspirine à dose anti-agrégante a étéindiquée chez $14 \%$ des patientes. 
L'évolutionétait favorable avec grossessemenée à terme chez 7 patientes (20\%), et la survenued'unerécurrenceou d'un décès dans 3 casrespectivement $(8,8 \%)$ [Tableau 2].

\section{Discussion:-}

Depuissa description princeps en 1983, le SAPL a tendance à êtrescindé, depuisces dix dernièresannées, en SAPL vasculaire versus SAPL obstétrical.

Ceci a étéavancé suite à plusieursconstats: les patientespeuventprésenter des thromboses vasculaires sans complications obstétricales et vice versa. Ainsi que le fait que la coexistence des deux manifestations ne représente que 2,5 voire $5 \%$ des casselon les séries [3]. Ce qui suggèreunephysiopathologiedifférente des deux présentations de la maladie.

Cependant, raressont les études dédiées à cetteentitéparticulière, responsable à la fois de complications obstétricales et maternelles. Dans leurregistreeuropéen, Alijotas et al. ontétudiél'évolution de 247 femmes présentant un SAPL obstétricalpur, leursdonnéesretrouvaient $53.8 \%$ de pertesfotales $<10 \mathrm{SA}$, suivies par $31.2 \%$ de fausses couches tardives> 10 SA [5].

Concernant la corrélation entre les anticorps anti-phospholipides et les évènementsobstétricaux, Alojitas et al. ontretrouvé la présenced'anticorps anti-cardiolipineisolés dans 66 cas. Ilsontretrouvéune forte corrélation entre la présenceisolée d'un anticoagulant lupique, de double positivitéou de triple positivité, et toutes les manifestations obstétricalesconfondues.

Plusieurs études ontconfirmécettecorrélation entre la présence d'un anticoagulant lupique circulant et le risquemajoré de survenue de complications obstétricales. Ainsi, dans son récent rapport, Yelnik a confirmé les données de l'étude prospective PROMISSE $(\mathrm{n}=144)$ sur un échantillon de 44 nouvellespatientes. Parmicelles qui avaient un anti-coagulant lupique circulant, $53 \%$ ontprésentéune complication obstétricale $[15 ; 16]$.

D’un autrecôté, aucunecorrélationn'aétéretrouvée pour les anti-cardiolipine et les anti-bêta 2 glycoprotéines [5].

Dans un autre travail, Opatrny et al. ontretrouvéunecorrélation positive entre les anti-cardiolipine et les fausses couches précoces $<13$ SA [6].

Les facteurs de risque de mauvaispronosticobstétrical qui ressortentsontreprésentéspar la présence d'un anticoagulant lupique circulant maisaussid'antécédent de thrombose systémique.

Concernant la priseen charge des patientesporteuses d'un SAPL obstétrical, elle se veut d'être multidisciplinaire, avec l'organisationd'une consultation pré-conceptionnelle, au cours de laquelle les différentes options thérapeutiques et leurseffetssecondairesdevraientêtreabordés. Il convientalors de classer les patientesselon le risque de survenue de complications obstétricalesenpatientes à hautrisque et patientes à faiblerisque. Le groupe à hautrisqueétantreprésenté par les patientesrépondant aux critèressuivants:présence de thrombose, anti-coagulant lupiquepositif, triple positivité, connectiviteassociée.

Deux groupes de patientespeuventégalementêtreindividualisés enfonction de leurtraitement: un premier groupe mis au préalable sous anti-coagulation curative du fait d'une thrombose antérieure, chez qui les antivitamine $\mathrm{K}$ doiventêtrearrêtésdès la découverte de la grossesse et l'anticoagulation curative poursuivie par unehéparine de bas poidsmoléculaire.

Le second groupe correspond aux patientesayantprésenté des fausses couches à répétition, sans évènementthrombotique. Les recommandationsactuellespréconisent de démarreruneaspirine à dose anti-agrégante 4 semainesavant la conception. Ce traitement sera renforcé au cours de la grossesse par l'adjonctiond'unehéparine à faible dose. Selonune revue Cochrane, cette association permettrait de réduire les pertesfotales de 54\% [12].

Encas de SAPL obstétricalréfractaire - définipar la survenue de pertesfoetales malgré un traitement par aspirine et HBPM bien conduit- l'escaladethérapeutique se fera par l'adjonction de corticoïdes, anti-paludéens de synthèse, échangesplasmatiques, immunoglobulines intra-veineuses, et plus récemment d'un anticorps monoclonal (rituximab) selon le degré de sévérité [1] 
L'évolutionglobale de nospatientesétaitsatisfaisante, aucundécèsni complication thrombotiquen'estsurvenu dans les formesobstétricalespures. Toutes les grossessesmenées sous association d'aspirine à faible dose et d'héparine de bas poidsmoléculaireontpuaboutir.

Le registreeuropéen a égalementretrouvé un excellent pronosticmaterno-fotallorsque le traitement a étéinitié. Avec un constatintéressant:près de $20 \%$ des patientes ne recevaient pas de traitementapproprié et seulement $30 \%$ ontreçuuneaspirine à faible dose avant la prochainegrossesse [5]. Cecisoulignel'intérêtd'améliorerl'approchethérapeutique.

Delesalle et al. ontrapporté des résultatssimilaires: dans leurcohorte, seulement 27 patientes (soit $57 \%$ ) ontbénéficié d'un traitementadapté. Sept grossessesontbénéficiéégalementd'unecorticothérapieorale et 11 d'hydroxychloroquine [4].

La survenue de récurrencesthrombotiquesouobstétricales, et de décès, a étéobservée chez les patientesprésentantuneformevasculaireassociée.

Ce pronostic plus sombre des formesthrombotiquesassociées a étéretrouvé dans l'étude de Bramham et al. qui ontincluunecohorte de 83 grossesses. Lorsqu'une thrombose étaitassociée, les patientesavaient plus souvent un lupus associé et le pronosticétaitégalementsignificativement plus mauvais [11].

Enfin, les données du registreeuropéenviennentconforterl'hypothèsed'uneentitéindividualisée, à pronosticdifférent du SAPL vasculaire, puisque les patientesprogressaientmoinsvers un lupus systémique, et présentaientmoins de complications thrombotiques [5].

Le syndrome des anti-phospholipidesestuneentitéparticulière, à risque de complications maternelles et aussifotales. Son diagnostic doitpousser à l'organisationd'unepriseen charge multidisciplinaire:

1. Programmationd'une consultation pré-conceptionnelle au cours de laquelle les différentsfacteurs de risquedoiventêtreindividualisés

2. Prescription d'uneaspirine à faible dose 4 semainesavant la conception

3. Adjonctiond'unehéparine à dose préventive pour un meilleurpronosticobstétrical

4. Dans les formesréfractaires (persistance de complications obstétricales malgré unepriseen charge optimale) adjonction de corticothérapie, d'héparine curative et d'hydroxychloroquine.

\section{Conclusion:-}

Le syndrome des anti-phospholipidesobstétricalestuneentité à partentière, caractérisée par un meilleurpronosticmaternel et un moindrerisque de thromboses systémiques.

Les donnéesactuelles ne permettent pas d'établir de recommandationsspécifiques de ce sous-groupe de patientes. Des études orientéesdevraientpermettre de mieuxcomprendre la physiopathologie de cette affection rare.

\section{Références:-}

1. Mekinian A, et al. Le syndrome des antiphospholipidesobstétrical: quelle est la place d'un traitementcomplémentaire à la combinaisonaspirine et héparine?GynécologieObstétriqueFertilité\&Sénologie (2017)

2. Miyakis S, Lockshin MD, Atsumi T, et al. International consensus statement on an update of the classification criteria for definite antiphospholipid syndrome (APS). J ThrombHaemost 2006;4(2):295-306

3. S. D'Ippolito, Obstetric Antiphospholipid Syndrome: A recent classification for an old defined disorder, Autoimmunity Reviews (2014)

4. Delesalle C, et al. Syndrome des anticorpsantiphospholipides et grossesse:pronosticobstétricalselon le type de SAPL. J GynecolObstetBiolReprod (Paris) (2014)

5. J.Alijotas-Reig et al. The European Registry on Obstetric Antiphospholipid Syndrome (EUROAPS): A survey of 247 consecutive cases. Autoimmunity Reviews 14 (2015)

6. Opatrny Le et al. Association between antiphospholipid antibodies and recurrent fetal loss in women without autoimmune disease: a metaanalysis. J Rheumatol 2006 
7. Committee on Practice Bulletins-Obstetrics, American College of Obstetricians and Gynecologists; Antiphospholipid syndrome. ACOG Practice Bulletin. ObstetGynecol 2012;120:1514-21

8. Wong LF et al. Recurrent early pregnancy loss andantiphospholipid antibodies: where do we stand? Lupus $2014 ; 23: 1226-8$.

9. Abou-Nassar $\mathrm{K}$ et al. The association between antiphospholipid antibodies and placenta-mediated complications: a systematic reviewand meta-analysis. Thromb Res 2011; 128:77-85.

10. Silver RM et al. Antiphospholipid antibodies and stillbirth. ObstetGynecol2013; 122:641-57.

11. Bramham $\mathrm{K}$ et al. Pregnancy outcome in different clinical phenotypes of antiphospholipid syndrome. Lupus (2010) 19, 58-64

12. Empson $\mathrm{M}$ et al. Prevention of recurrent miscarriage for women with antiphospholipid antibody or lupus anticoagulant. Cochrane Database Syst Rev 2005; CD002859

13. Garba Abdullahi Z. et al. Antiphospholipid antibodies among pregnant women with recurrent fetal wastage in a tertiary hospital in Northern Nigeria. Ann Afr Med. 2016 Jul-Sep; 15(3): 133-137

14. Fredi M. et al. Risk Factors for adverse Maternal and Fetal Outcomes in Women With confirmed aPl Positivity: results From a Multicenter study of 283 Pregnancies. Front. Immunol. 9:864

15. Yelnik M. et al. Lupus anticoagulant is the main predictor of adverse pregnancy outcomes in aPL-positive patients: validation of PROMISSE study results. Lupus Science \& Medicine 2016;3: e000131. doi:10.1136/lupus-2015-000131

16. Lockshin M.D. e al. Lupus anticoagulant, but not anticardiolipin antibody, predicts adverse pregnancy outcome in patients with antiphospholipid antibodies. Arthritis Rheum. 2012 July; 64(7): 2311-231

17. Akhlaghi F. Study on Antiphospholipid/Anticardioliplin Antibodies in Women with Recurrent Abortion

18. Cadavid AP (2017) Aspirin: The Mechanism of Action Revisited in the Context of Pregnancy Complications. Front. Immunol. 8:261. doi: 10.3389/fimmu.2017.00261. 